\section{Original Article}

Check for updates

\section{OPEN ACCESS}

Received: Jun 29, 2019

Revised: Jul 26, 2019

Accepted: Jul 29, 2019

Correspondence to

Luo Zhang, MD, PhD

Department of Otolaryngology Head and Neck

Surgery, Beijing TongRen Hospital, Capital

Medical University, No. 1, DongJiaoMinXiang

Street, DongCheng District, Beijing 100730,

China.

Tel: +86-139-1083-0399

Fax: +86-10-85115988

E-mail: dr.luozhang@139.com

\section{Chengshuo Wang, MD, PhD}

Department of Otolaryngology Head and Neck Surgery, Beijing TongRen Hospital, Capital Medical University, No. 1, DongJiaoMinXiang Street, DongCheng District, Beijing 100730,

China.

Tel: +86-139-1162-3569

Fax: +86-10-85115988

E-mail:wangcs830@126.com

†Mu Xian and Siyuan Ma contributed equally to this work.

Copyright (C 2020 The Korean Academy of Asthma, Allergy and Clinical Immunology . The Korean Academy of Pediatric Allergy and Respiratory Disease

This is an Open Access article distributed under the terms of the Creative Commons Attribution Non-Commercial License (https:// creativecommons.org/licenses/by-nc/4.0/) which permits unrestricted non-commercial use, distribution, and reproduction in any medium, provided the original work is properly cited.

\title{
Particulate Matter 2.5 Causes Deficiency in Barrier Integrity in Human Nasal Epithelial Cells
}

\author{
Mu Xian $\left(\mathbb{1},{ }^{1,2+}\right.$ Siyuan Ma $\mathbb{D}^{1,2+}$ Kuiji Wang $\mathbb{1},^{1,2}$ Hongfei Lou $\mathbb{1}^{1,2}$ Yang Wang $\mathbb{D}^{, 2}$ \\ Luo Zhang $\left(\mathbb{D},{ }^{1,2^{*}}\right.$ Chengshuo Wang $\mathbb{1}^{1,2^{*}}$ Cezmi A. Akdis $\mathbb{1}^{3,4}$
}

'Department of Otolaryngology Head and Neck Surgery, Beijing TongRen Hospital, Capital Medical University, Beijing, China

${ }^{2}$ Beijing Key Laboratory of Nasal Diseases, Beijing Institute of Otolaryngology, Beijing, China ${ }^{3}$ Swiss Institute of Allergy and Asthma Research (SIAF), University of Zurich, Davos, Switzerland ${ }^{4}$ Christine Kühne-Center for Allergy Research and Education, Davos, Switzerland

\section{ABSTRACT}

Purpose: The effect of air pollution-related particulate matter (PM) on epithelial barrier function and tight junction (TJ) expression in human nasal mucosa has not been studied to date. This study therefore aimed to assess the direct impact of PM with an aerodynamic diameter less than $2.5 \mu \mathrm{m}$ (PM2.5) on the barrier function and TJ molecular expression of human nasal epithelial cells.

Methods: Air-liquid interface cultures were established with epithelial cells derived from noninflammatory nasal mucosal tissue collected from patients undergoing paranasal sinus surgery. Confluent cultures were exposed to 50 or $100 \mu \mathrm{g} / \mathrm{mL}$ PM2.5 for up to 72 hours, and assessed for 1) epithelial barrier integrity as measured by transepithelial resistance (TER) and permeability of fluorescein isothiocyanate (FITC) $4 \mathrm{kDa}$;2) expression of TJs using real-time quantitative polymerase chain reaction and immunofluorescence staining, and 3) proinflammatory cytokines by luminometric bead array or enzyme-linked immunosorbent assay. Results: Compared to control medium, 50 and/or $100 \mu \mathrm{g} / \mathrm{mL}$ PM2.5-treatment 1) significantly decreased TER and increased FITC permeability, which could not be restored by budesonide pretreatment; 2) significantly decreased the expression of claudin-1 messenger RNA, claudin-1, occludin and ZO-1 protein; and 3) significantly increased production of the cytokines interleukin-8, TIMP metallopeptidase inhibitor 1 and thymic stromal lymphopoietin. Conclusions: Exposure to PM2.5 may lead to loss of barrier function in human nasal epithelium through decreased expression of TJ proteins and increased release of proinflammatory cytokines. These results suggest an important mechanism of susceptibility to rhinitis and rhinosinusitis in highly PM2.5-polluted areas.

Keywords: Epithelial cells; particulate matters; nasal mucosa; rhinitis; asthma; cytokines

\section{INTRODUCTION}

Airway epithelium plays a critical role in forming a physical barrier, which protects the submucosal tissues from harmful substances inhaled. This barrier function is primarily determined by the integrity of intercellular junctions, which consist of the apical tight junctions (TJs) and underlying adherent junctions (AJs). TJs and AJs establish cell-cell contact 
ORCID iDs

Mu Xian (iD)

https://orcid.org/0000-0003-4153-6314

Siyuan Ma iD

https://orcid.org/0000-0002-5075-6792

Kuiji Wang (iD)

https://orcid.org/0000-0002-0052-6278

Hongfei Lou (iD

https://orcid.org/0000-0002-8553-812X

Yang Wang in

https://orcid.org/0000-0003-1231-7278

Luo Zhang (iD)

https://orcid.org/0000-0002-0910-9884

Chengshuo Wang (iD

https://orcid.org/0000-0003-0646-5135

Cezmi A. Akdis (iD)

https://orcid.org/0000-0001-8020-019X

Disclosure

There are no financial or other issues that might lead to conflicts of interest. and cell polarity, and regulate the paracellular movement of ions and macromolecules. ${ }^{1}$ Recent studies have documented that TJ function is defective or altered in multiple airway diseases such as chronic rhinosinusitis, ${ }^{2}$ allergic rhinitis, ${ }^{3}$ chronic obstructive pulmonary disease $^{4}$ and asthma. ${ }^{5}$

Epidemiological studies ${ }^{6-8}$ suggest that exposure to escalating environmental particulate matter (PM) is associated with an increase in respiratory tract diseases, with 1 recent study indicating that airborne PM may directly cause airway epithelial cell injury. ${ }^{9}$ Dysfunctional epithelial barrier may enhance uptake or exposure of deeper tissues to foreign pathogens, irritants, pollutants and allergens, and can lead to higher activation of subepithelial cells, such as immune cells. ${ }^{4,10}$ Thus, chronic exposure to airborne PM may potentially predispose an individual to chronic mucosal inflammatory disease by impairing airway barrier function. However, the causal relationship between PM and airway epithelial TJ dysfunction is not clear.

The aim of the present study was to assess the direct impact of PM with an aerodynamic diameter less than $2.5 \mu \mathrm{m}$ (PM2.5) on the function and expression pattern of normal human nasal epithelial TJ. Nasal mucosa biopsy specimens obtained from noninflammatory subjects exhibited lower TJs expression in a higher PM2.5 season (i.e., winter) compared to those obtained in the summer. Using air-liquid interface (ALI) cultures of noninflammatory human nasal epithelial cells (NECs), we evaluated whether PM2.5 induces leakiness in epithelial barrier, along with down-regulation of claudin-1 at both messenger RNA (mRNA) and protein level, and whether it induces production of proinflammatory cytokines, which may cause TJs damage and tissue remodeling in the nasal mucosa.

\section{MATERIALS AND METHODS}

\section{Patients}

Patients undergoing paranasal sinus surgery for noninflammatory conditions (i.e., cerebrospinal fluid leak, bullous middle turbinate and septal deformities) were enrolled in the study. Surgery was conducted under general anaesthesia, and nasal mucosa was obtained from the middle turbinate or the uncinate process according to different purposes of the operation. Patients with histories suggestive of allergies or with total immunoglobulin E (IgE) levels exceeding $100 \mathrm{kU} / \mathrm{L}$ or allergen-specific IgE level > $3.51 \mathrm{kU} / \mathrm{L}$ were excluded. All patients were nonsmokers. The study protocol was approved by the Ethics Committee of Beijing Tongren Hospital, and all patients provided written informed consent prior to any samples being taken for investigation.

For in vivo study, the mucosal samples were obtained from our sample bank of patients meeting the above requirements and operated in the summer (i.e., June and July) or winter (i.e., January and February) months. One part of the mucosa was stored at $-80^{\circ} \mathrm{C}$ for future RNA isolation, and the other was embedded in $4 \%$ paraffin for histological evaluation.

\section{Isolation and ALI cultures of primary NECs}

Freshly obtained nasal mucosal samples were washed in phosphate buffer saline (PBS) with $200 \mathrm{U} / \mathrm{mL}$ penicillin and $50 \mathrm{mg} / \mathrm{mL}$ streptomycin and were then enzymatically digested in 0.1\% pronase (Protease XIV; Sigma-Aldrich, St. Louis, MO, USA) solution in Dulbecco's modified eagle media (DMEM) culture medium supplemented with $100 \mathrm{U} / \mathrm{mL}$ penicillin. After overnight incubation at $4^{\circ} \mathrm{C}$ with gentle shaking, the protease reaction was stopped by 
addition of $10 \%$ fetal bovine serum. Cells were washed in culture medium and pelleted by centrifugation at $100 \mathrm{~g}$ for 5 minutes. The cell pellet was then resuspended in $10 \mathrm{~mL}$ of culture medium and incubated in a plastic culture flask at $37^{\circ} \mathrm{C}$ for 1 hour to remove fibroblasts.

Freshly isolated NECs were seeded on $0.4-\mu \mathrm{m}, 0.33-\mathrm{cm}^{2}$ polyester transwell inserts (Costar, Corning, NY, USA) at a density of $10^{5}$ cells per transwell in DMEM/F12: BEGM (1:1) culture medium (Lonza, Walkersville, $\mathrm{MD}$, USA) and incubated at $37^{\circ} \mathrm{C}$ in $5 \% \mathrm{CO}_{2}$ in air atmosphere. The culture medium was changed every other day, until the NECs grew to complete confluence, at which stage the apical culture medium was removed and the cells were allowed to differentiate further as ALI cultures.

\section{Purification and characterisation of PM2.5}

PM2.5 was a gift from the Institute for Environmental Health and Related Product Safety, Chinese Center for Disease Control and Prevention. The PM2.5 samples were collected on specialized filters in urban areas of Beijing during the period of 20 October to 25 November, 2014. Particles were released from filters and dispersed in solution by ultra-sonication in endotoxin-free $0.9 \%$ saline for 30 minutes. After being dried, the PM2.5 samples were weighed and aliquoted in PBS before being stored at $-20^{\circ} \mathrm{C}$ until required. The assessment methods and results of the chemical composition of PM2.5 are presented in Supplementary Table S1.

\section{Assessment of PM2.5 cytotoxicity to human cultured NECs}

Cytotoxicity of PM2.5 was assessed by measuring lactate dehydrogenase (LDH) released into the culture medium of human NECs incubated in the presence of increasing concentrations of PM2.5 for 24 hours. The 50- $\mu \mathrm{L}$ aliquots of media were transferred to a 96 well plate and mixed with $50-\mu \mathrm{L}$ reaction mixture (ThermoFisher scientific, Woburn, MA, USA). Thirty minutes after incubation in the dark at room temperature, the absorbance of the formazan salt was measured at $492 \mathrm{~nm}$. Absorbance at $620-\mathrm{nm}$ wavelength was used as a reference. All measurements were made in triplicates and cytotoxicity was calculated according to the manufacturer's instructions, including a background control for LDH activity in the media, a control for untreated cells ( $0 \%$ lysis of the cells) and a control for maximum LDH release $(100 \%$ lysis of the cells).

\section{Assessment of the effect of PM2.5 on transepithelial resistance (TER) and paracellular flux in NECs established as ALI cultures}

Based on LDH cytotoxicity experiments, PM2.5 concentrations less than $100 \mu \mathrm{g} / \mathrm{mL}$ were considered nontoxic and applied to ALI cultures. The experiments were initiated at about 3 weeks in ALI culture, when TER reached a plateau of above $300 \Omega \times \mathrm{cm}^{2}$. To mimic the in vivo state in which the pollution component would come into contact with the ciliated airexposed surface of the respiratory epithelium, NECs were exposed to $50 \mu \mathrm{g} / \mathrm{mL}$ or $100 \mu \mathrm{g} / \mathrm{mL}$ PM2.5 added to the apical compartment for 8 hours daily. Then PM2.5 was sucked away and the cells were rinsed 3 times with PBS. Control cultures were prepared by adding only culture medium to the apical compartment.

Similarly, the effect of budesonide, an intranasal steroid commonly used as first-line therapy for nasal inflammation, was investigated by adding $1 \mu \mathrm{mol} / \mathrm{L}$ budesonide (Sigma-Aldrich) or culture medium into the upper chamber for 1 hour prior to treatment with PM2.5. The concentration of budesonide was adapted from the study of Sekiyama et al., ${ }^{11}$ who claimed that $1 \mu \mathrm{mol} / \mathrm{L}$ budesonide could directly promote the permeability barrier function of airway epithelial cells Calu-3. 
TER and paracellular diffusion of fluorescein isothiocyanate (FITC)-labeled 4-kDa dextran (Sigma-Aldrich) were determined as surrogate markers of epithelial integrity. TER measurements were made at 0 (before stimulation), 24, 48 and 72 hours after incubation with PM2.5, using a Millicell-ERS Volt-Ohm Meter (Millipore, Temecula, CA, USA). Cultures with TER $<300 \Omega \times \mathrm{cm}^{2}$ were not included in experiments (14\%), and TER was measured in triplicate for each subject.

Paracellular flux measurements were made after TER experiments. Paracellular permeability was evaluated by adding of $2 \mathrm{mg} / \mathrm{mL}$ FITC-dextran to the apical surface of the ALI cultures and then measuring the intensity of FITC in the basolateral medium after 12 hours, with an ELISA reader (Mithra LB 940; Berthold Technologies, Bad Wildbad, Germany) at $480 \mathrm{~nm}$. Experiments were conducted with cells from each participant, employing a minimum of 2 cultures established in multiple well plates.

\section{Quantitative real-time polymerase chain reaction (PCR) and immunostaining of TJs}

After incubation with PM2.5 for 72 hours, NEC monolayers were dissected from transwell membranes. Total RNA was extracted and purified with an RNeasy Mini Kit (Qiagen, Hilden, Germany) and RNase-free DNase (Qiagen), according to the manufacturer's instructions. RNA was reverse transcribed into complementary DNA using a Revert Aid RT kit containing random hexamers (ThermoFisher Scientific) and quantitative real-time PCR was performed on a Bio-Rad iCycler using the iQ SYBR Green Supermix (BioRad, Hercules, CA, USA) as described in the manufacturer's protocol. Sequences of the predesigned primers used are listed in Supplementary Table S2. The amount of elongation factor $1 \alpha$ mRNA was used to standardize the quantities of each transcript according to the $2^{-\triangle \Delta C T}$ value.

A similar immunofluorescent staining procedure was employed for both paraffin-embedded biopsy sections and ALI cultures. Detailed information on the methods employed for staining TJs (claudin-1, ZO-1 and occludin) is described in the Supplementary Data S1. The fluorescence intensities of acquired images were quantified using ImageJ software (National Institute of Health, Bethesda, MD, USA).

\section{Luminex and enzyme-linked immunosorbent assay (ELISA) analysis of cytokine concentration in culture medium}

Inflammatory cytokines were measured in culture medium in the basolateral chamber collected after measurement of TER at the end of the 72-hour incubation period in the presence of PM2.5, and before addition of FITC into the upper chamber. All samples were stored in aliquots at $-20^{\circ} \mathrm{C}$ until analysis for interleukin (IL)-8, IL-25, IL-33, transforming growth factor (TGF)- $\beta$, matrix metalloproteinase (MMP)-9, TIMP metallopeptidase inhibitor 1 (TIMP-1) and thymic stromal lymphopoietin (TSLP). Samples were assessed in duplicate and all samples, apart from IL-8 and TSLP, were analyzed using the Human Magnetic Luminex Screening Assay (R\&D, Santa Clara, CA, USA) according to the manufacturer's recommendations. The assay was performed using an 8-point standard curve for each cytokine. Samples were analysed on a Bio-Plex 200 System (BioRad), and the data were evaluated using the Bio-Plex Manager software (BioRad). Standards, internal controls and samples are reported as means of duplicate measurements. IL-8 and TSLP were assayed by commercial ELISA kits (R\&D) according to the manufacturer's instructions. 


\section{Statistical analysis}

Data were analyzed using GraphPad Prism 5 software (GraphPad Software, La Jolla, CA, USA). Data are presented as means \pm standard errors. Differences between groups were analyzed using the 2-tailed unpaired $t$ test or the Mann-Whitney $U$ test. Two-way analysis of variance or the Kruskal-Wallis test with post hoc analysis was used to compare multiple groups. Values of $P<0.05$ were considered statistically significant.

\section{RESULTS}

\section{TJ expression in nasal mucosa harvested in different seasons}

Five and 7 samples of noninflammatory nasal mucosa were collected during endoscopic surgery in the summer (i.e., June and July) and winter (i.e., January and February), respectively. For quantitative PCR [qPCR] experiment, sample numbers were 5 for both winter and summer. The clinical data of these patients, including average 24-hour PM2.5 concentration in the week prior to the surgery, are shown in Supplementary Table $\mathbf{3} 3$.

To visualize TJ expression in the nasal mucosa in different seasons, paraffin-embedded biopsy sections were stained for claudin-1, occludin and ZO-1 (Fig. 1). The immunofluorescent staining for ZO-1 and occludin was mainly confined to the upper most level of the epithelium, whereas the immunofluorescent staining for claudin-1 was distributed more extensively in the epithelium. The expression pattern of TJs was consistent with those found in other studies that claudin-1 was expressed not only pericellularly but in the cytoplasm, while ZO-1 and occludin were almost exclusively confined to the membrane. The cytoplasmic distribution of claudin-1 was consistent with those of previous studies. ${ }^{12,13}$ Furthermore, the immunoreactivity of claudin-1, ZO-1 and occludin was significantly higher in the summer samples than in the winter samples. The qPCR of these samples showed that the arbitrary unit of TJ mRNAs (including ZO-1, ZO-2, CLDN-1, CLDN-4, CLDN-7 and occludin) all displayed a tendency for lower expression during the winter than during the summer; however, the differences were not statistically significant (Supplementary Fig. S1).

\section{Effect of PM2.5 on NECs established as ALI cultures}

After visualization of TJ expression in different seasons, we aimed to assess the impact of PM2.5 in cultured NECs. ALI cultures of NECs were established from 23 samples of noninflammatory nasal mucosa. Assessment of cytotoxicity of PM2.5 to NECs demonstrated that PM2.5 concentrations above $100 \mu \mathrm{g} / \mathrm{mL}$ led to prominent cell death (more than $10 \%$ ) and was therefore considered toxic (Supplementary Fig. S2). Concentrations of $50 \mu \mathrm{g} / \mathrm{mL}$ and 100 $\mu \mathrm{g} / \mathrm{mL}$ were used in all subsequent experiments with ALI cultures.

Incubation of ALI cultures with $50 \mu \mathrm{g} / \mathrm{mL}$ PM2.5 intermittently for 72 hours significantly decreased the relative TER (ratio of each time point TER to that of 0 hour) compared to baseline level $(1.02 \pm 0.10$ vs. $0.73 \pm 0.07, P<0.01)$, whereas incubation with $100 \mu \mathrm{g} / \mathrm{mL}$ PM2.5 significantly decreased relative TER by 24 hours $(0.99 \pm 0.06$ vs. $0.73 \pm 0.06, P<0.01)$. Furthermore, the effect of $100 \mu \mathrm{g} / \mathrm{mL}$ PM2.5 on TER was greater than that of $50 \mu \mathrm{g} / \mathrm{mL}$ PM2.5 at each time point (Fig. 2A), indicating that PM2.5 lowered TER in a concentration- and timedependent manner. According to our recovery study (Supplementary Fig. S3), after PM2.5 was withdrawed for 96 hours, relative TER returned to the baseline level. Since it took about 3 weeks to setup an ALI culture, we assumed the time point of tissue harvest would not affect the barrier integrity of cultured NECs. 

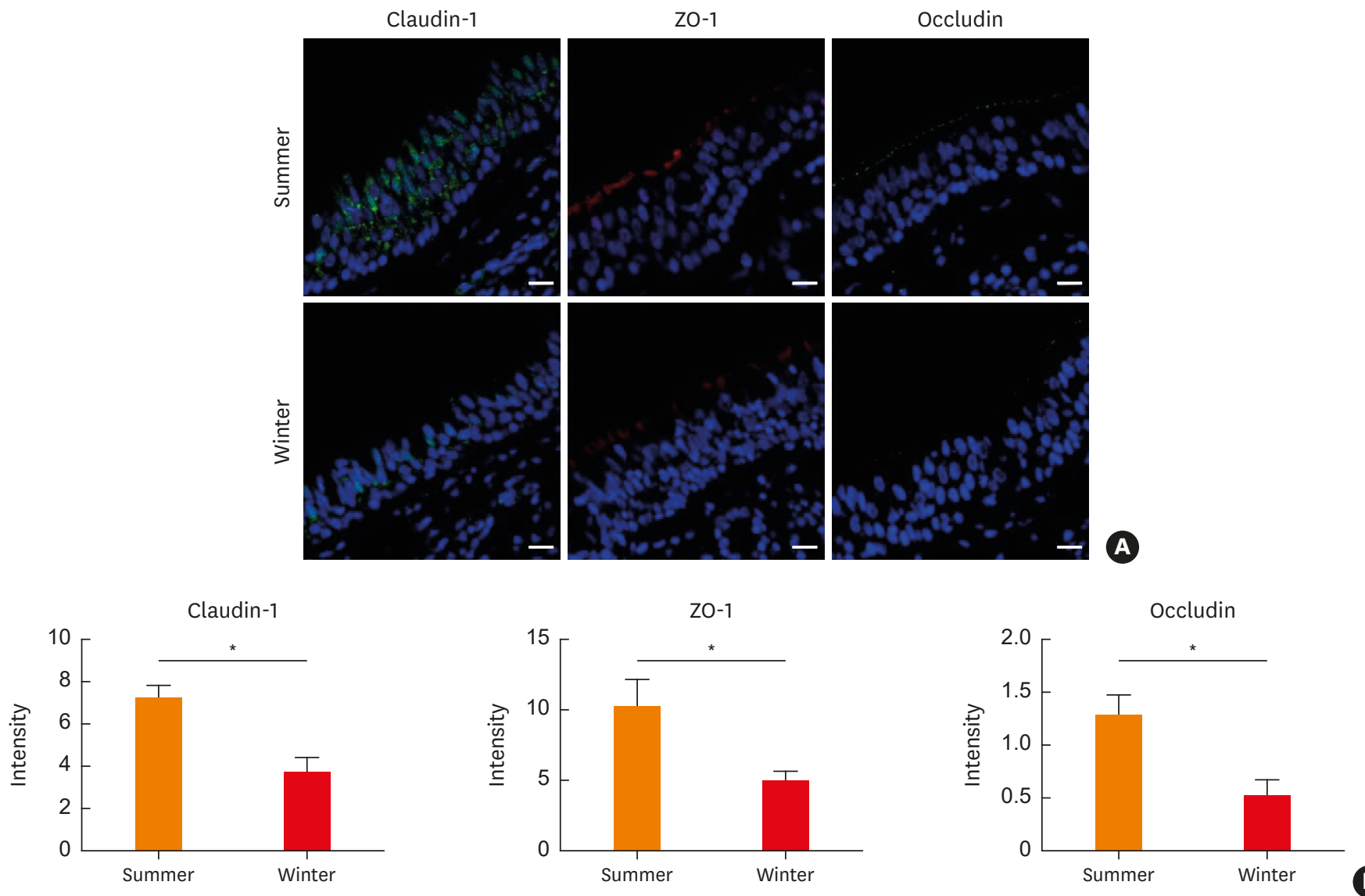

Fig. 1. (A) Representative images of immunofluorescent-stained claudin-1, ZO-1 and occludin in noninflammatory nasal mucosa collected during different seasons. The immunofluorescent staining for ZO-1 (red) and occludin (green) was mainly restricted to the apical compartment of epithelium, whereas the distribution of claudin-1 (green) was more extensive in the epithelium. A relatively weak staining was seen for claudin-1, occludin and ZO-1 in biopsy specimens collected during the winter compared to specimens collected during the summer. Bar $=20 \mu \mathrm{m}$. (B) Fluorescence intensities were quantified using ImageJ software (winter vs. summer; $\mathrm{n}=7$ and 5 , respectively).

${ }^{*}$ Statistical significance at a level of $P<0.05$. 

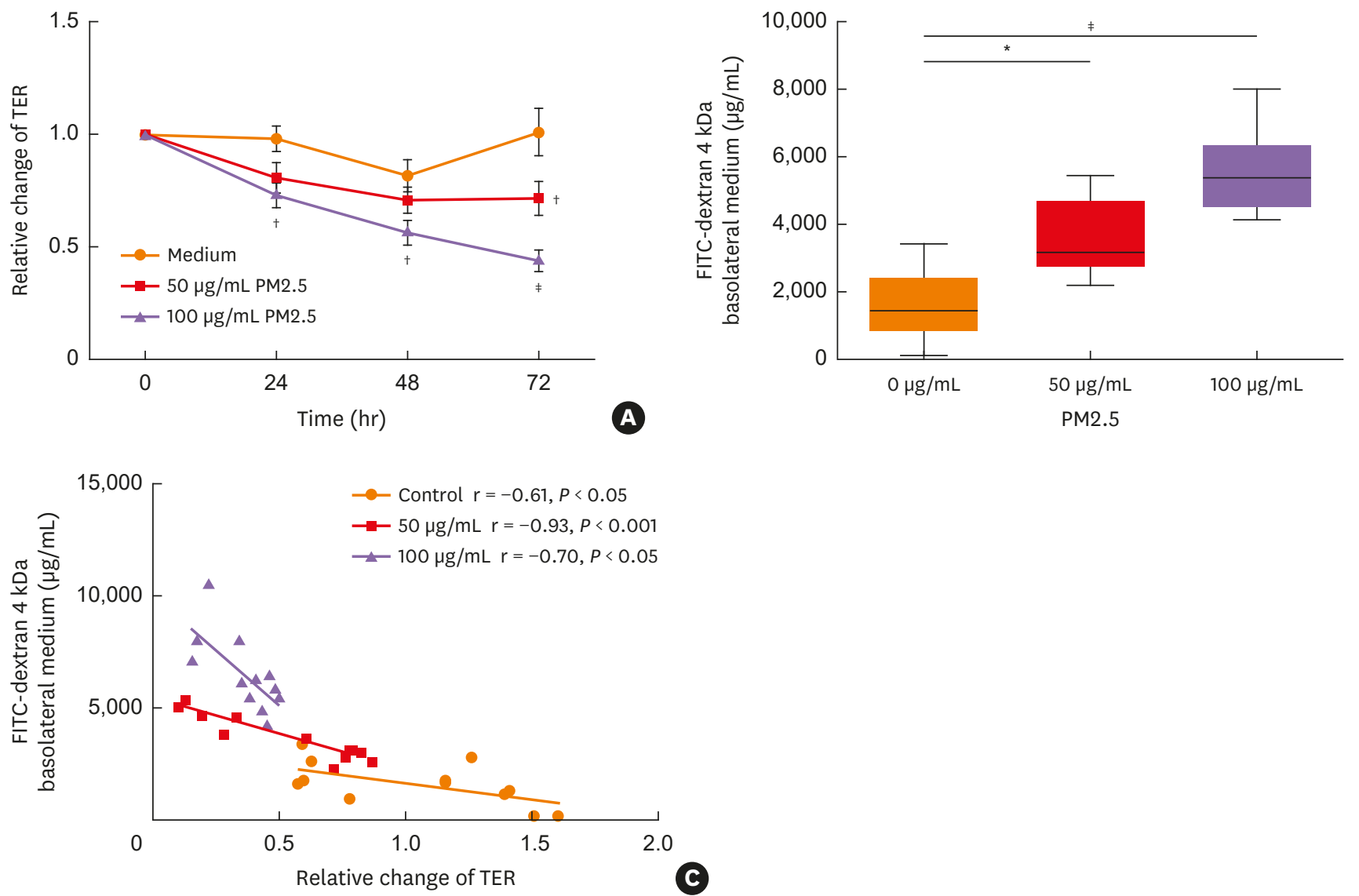

Fig. 2. Effect of PM2.5 on the barrier function of ALI cultures established from NECs. (A) TER at different time points following exposure to medium alone (control), $50 \mu \mathrm{g} / \mathrm{mL}$ PM2.5 or $100 \mu \mathrm{g} / \mathrm{mL}$ PM2.5 intermittently. Due to some inherent variability in baseline TER measurements across samples, all measurements were normalized to a value of 1.0 at the beginning of the measurement (O hour). (B) Dose-dependent increase in FITC-dextran paracellular flux in ALI cultures treated with different doses of PM2.5. Barrier integrity was determined by paracellular flux in response to PM2.5 treatment after 72 hours. (C) TER negatively correlates with FITC-dextran permeability in ALI cultures (exposed to 100 and $50 \mu \mathrm{g} / \mathrm{mL}$ PM2.5 or medium alone). Data are expressed as mean \pm standard error of the mean, $\mathrm{n}=12$ donors per group in duplicate.

PM2.5, particulate matter with an aerodynamic diameter of less than $2.5 \mu \mathrm{m}$; FITC, fluorescein isothiocyanate; ALI, air-liquid interface; TER, transepithelial resistance. ${ }^{*} P<0.05 ;{ }^{\dagger} P<0.01 ;{ }^{\ddagger} P<0.001$

\section{Effect of PM2.5 treatment on TJs mRNA and protein expression in ALI cultures established from NECs}

The expression of TJ genes previously shown to be detected in relatively high levels in human nasal mucosa was investigated, including claudin-1, claudin-4, claudin-7, occludin, ZO-1 and $\mathrm{ZO}-2 .{ }^{17,18}$

Incubation with $100 \mu \mathrm{g} / \mathrm{mL}$ PM2.5 significantly increased the expression of claudin-7 and ZO-1 mRNAs, while the expression of claudin-1 in ALI cultures decreased (Fig. 4). Incubation with $50 \mu \mathrm{g} / \mathrm{mL}$ PM2.5 affected the mRNA expression of claudin-7 and claudin- 1 similarly, but did not alter the expression of ZO-1 mRNA.

Assessment of the effects of PM2.5 on the expression of TJ proteins by confocal fluorescence microscopy demonstrated that fluorescence signals were interrupted and discontinuous at perijunctional areas in PM2.5-treated cultures, while claudin-1, occludin and ZO-1 presented as continuous fluorescent ring signals at the intercellular junction in untreated control 

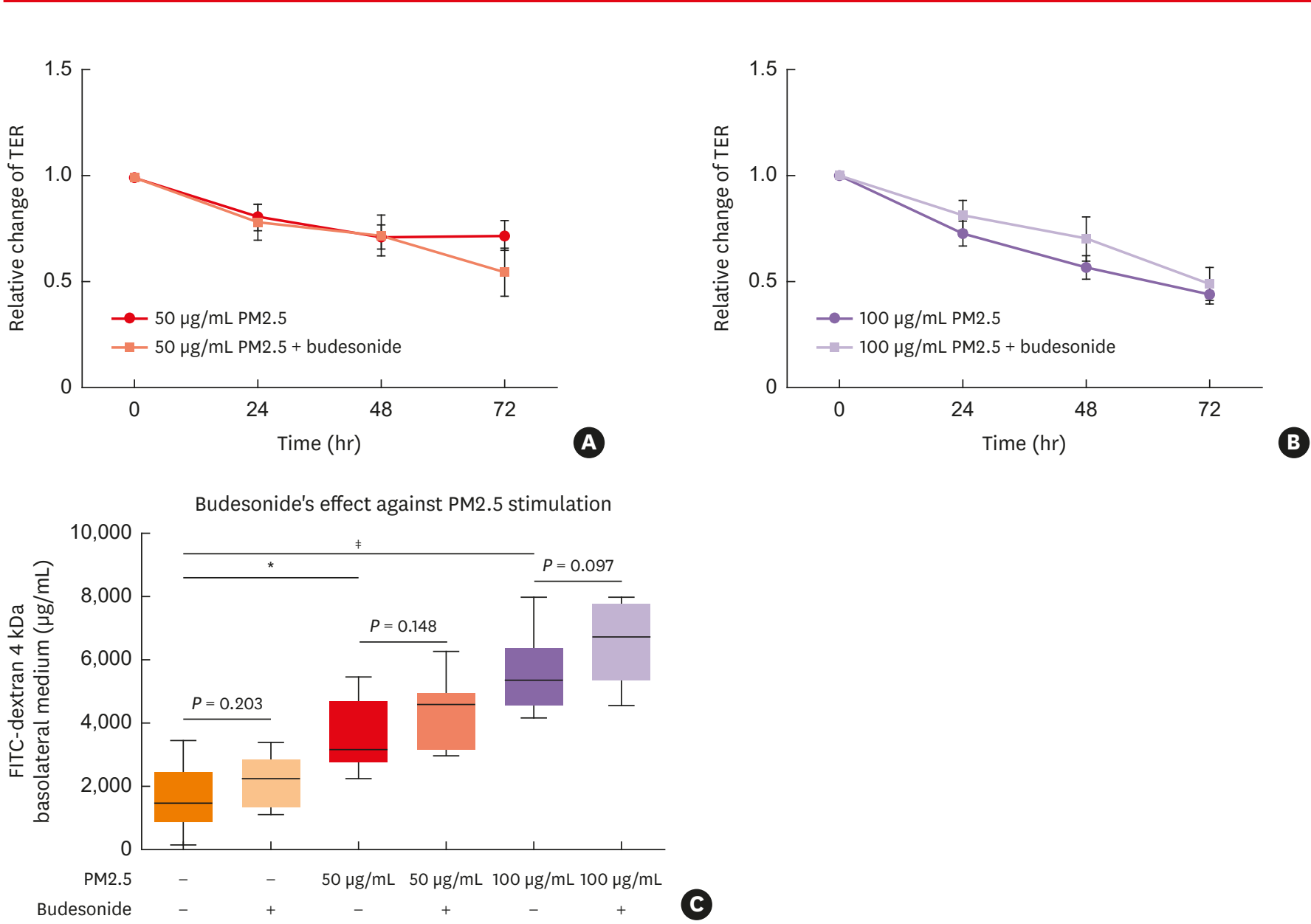

Fig. 3. PM2.5 undermined the barrier integrity of air-liquid interface cultures established from nasal epithelial cells, which could not be prevented by budesonide pretreatment. The relative TER of cultures exposed to $50 \mu \mathrm{g} / \mathrm{mL}$ PM2.5 (A) or $100 \mu \mathrm{g} / \mathrm{mL}$ PM2.5 (B) and (C) paracellular flux of FITC-dextran $4 \mathrm{kDa}$ at 72 hours in cultures incubated in the absence or presence of $50 / 100 \mu \mathrm{g} / \mathrm{mL}$ PM2.5 was not significantly altered by pretreatment of the cultures for 1 hour with budesonide (1 $\mu \mathrm{mol} / \mathrm{L})$. Data are expressed as mean \pm standard error of the mean, $\mathrm{n}=5$ donors per group in duplicate.

PM2.5, particulate matter with an aerodynamic diameter less than $2.5 \mu \mathrm{m}$; FITC, fluorescein isothiocyanate; TER, transepithelial resistance.

${ }^{*} P<0.05 ;{ }^{\dagger} P<0.001$. 

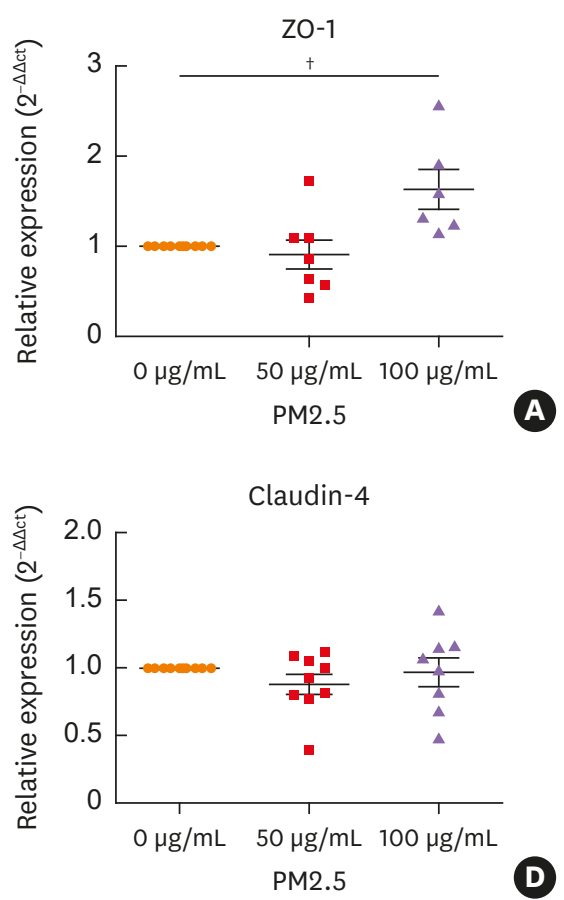

(-

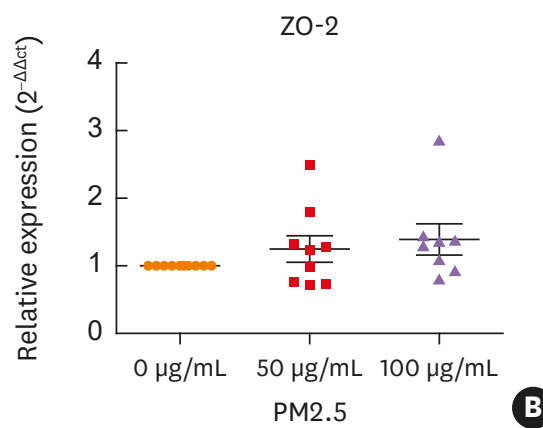

B
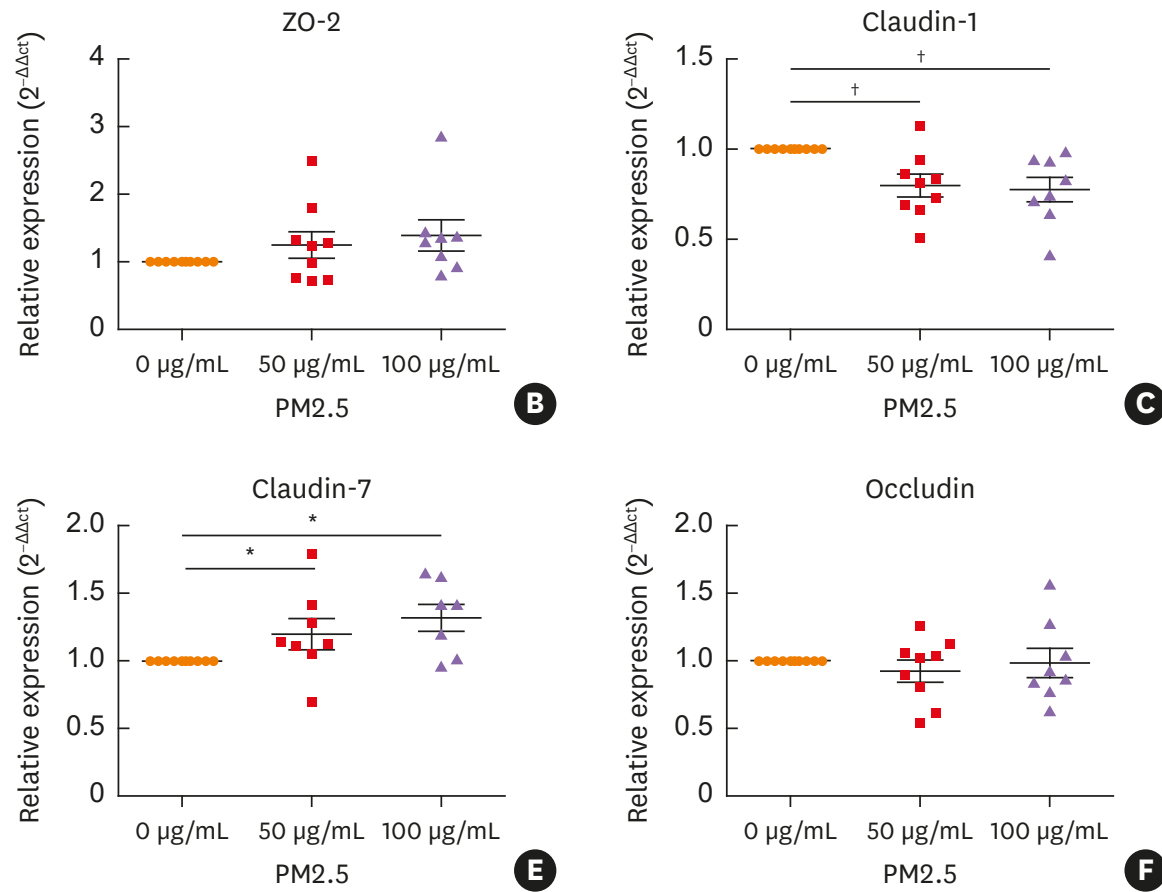

Fig. 4. Effect of PM2.5 on the expression of tight junction mRNAs in air-liquid interface cultures established from nasal epithelial cells. After incubation with PM2.5 for 72 hours, relative mRNA expression of claudin-1 was significantly decreased and expression of ZO-1 and claudin-7 significantly increased in cultures treated with $100 \mu \mathrm{g} / \mathrm{mL}$ PM2.5, compared to control cultures $(\mathrm{n}=10$ each for the control and 50- $\mu \mathrm{g} / \mathrm{mL}$ PM2.5-treated groups, $\mathrm{n}=9$ for the 100 - $\mu \mathrm{g} / \mathrm{mL} \mathrm{PM} 2.5$ treated group).

PM2.5 with an aerodynamic diameter of less than $2.5 \mu \mathrm{m}$; mRNA, messenger RNA.

${ }^{*} P<0.05,{ }^{\dagger} P<0.01$.

\section{DISCUSSION}

Intercellular TJs are important components for efficient epithelial barrier function: the first-line defence against invasion by environmental pathogens and entrance of antigens. TJs form homodimer bands that prevent cells and proteins passing through the epithelium, and regulate paracellular water permeability selectively. ${ }^{19}$ Several studies have shown PMinduced reductions in barrier functions of skin, ${ }^{20}$ blood-brain barrier, ${ }^{21}$ endothelial cells ${ }^{22}$ and gastrointestinal tract ${ }^{23}$; however, little research has focused on the impact of PM on especially the integrity of upper airway epithelial thus far.

To understand the relationship between PM2.5 and increased nasal inflammation, the present study investigated the function and expression of TJs in NECs ALI cultures established from the nasal samples of normal human subjects. The findings of increasing concentrations of LDH leakage from cells supported the notion that cytotoxicity of PM2.5 is correlated with its concentration. While concentrations above were toxic, increased cell death and resulted in substantial loss of epithelial barrier integrity, concentrations below 100 $\mu \mathrm{g} / \mathrm{mL}$ PM2.5 were much less toxic but still led to a significantly defective barrier function as well as decreased expression of TJ mRNAs and proteins. Less expression of TJ proteins highlighted by immunofluorescence staining was also shown in nasal mucosa biopsy specimens obtained from non inflammatory subjects during the winter compared to those obtained in the summer. Ambient PM2.5 concentrations in Beijing are much higher in the winter as a result of additional heating requirements than in the summer. ${ }^{24}$ Besides PM2.5 concentration, other meteorological factors, including humidity and temperature, vary in 

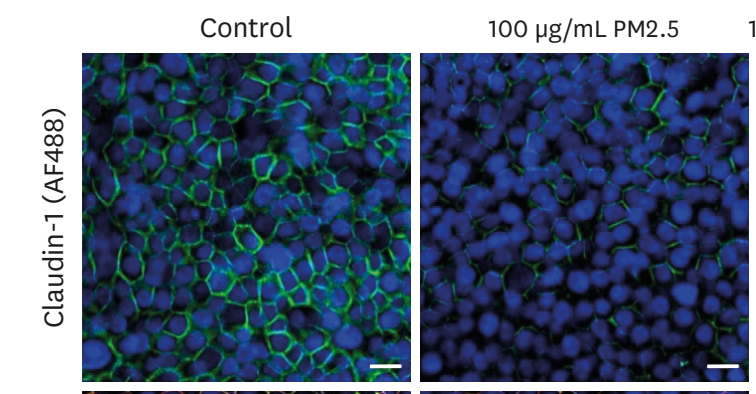

$100 \mu \mathrm{g} / \mathrm{mL}$ PM2.5 + budesonide
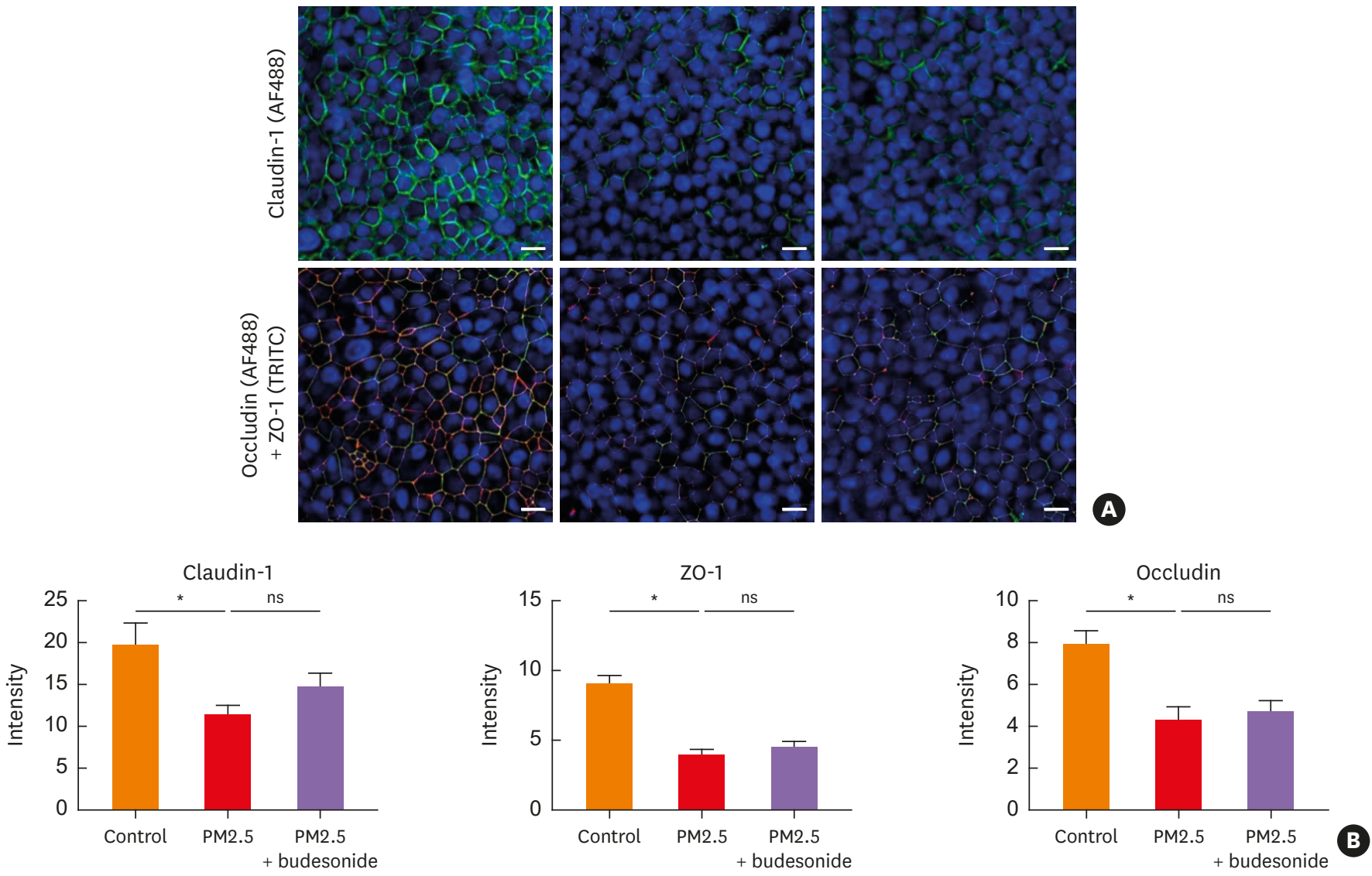

Fig. 5. (A) Representative images of claudin-1 (green), occludin (green) and ZO-1 (red) immunofluorescent staining in cultured nasal epithelial cells exposed to medium alone, $100 \mu \mathrm{g} / \mathrm{mL}$ PM2.5 or $100 \mu \mathrm{g} / \mathrm{mL}$ PM2.5 pretreated with budesonide. Cultures exposed to medium alone displayed distinct TJs at cell borders, whereas cultures treated with PM2.5 displayed diminished and less discernible TJs at cell-cell junctions. Cultures pre-treated with budesonide showed similar changes to those treated with just PM2.5. Representative images from 3 donors each are shown. Bar $=20 \mu \mathrm{m}$. (B) Fluorescence intensities were quantified using Image J software.

PM2.5, particulate matter with an aerodynamic diameter less than $2.5 \mu \mathrm{m}$; N.S., not significant; TJ, tight junction; TRITC, tetramethylrhodamine. ${ }^{*} P<0.05$. 

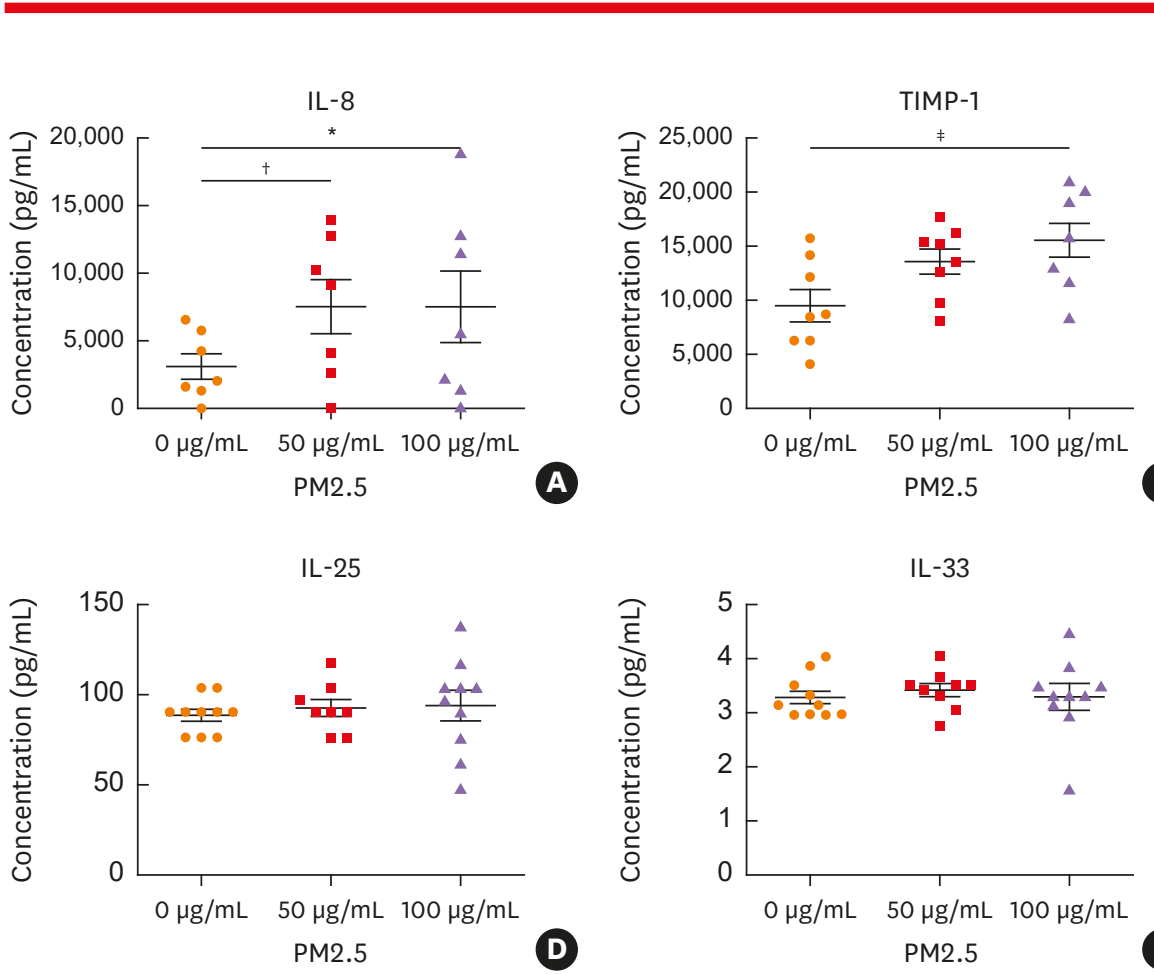

D

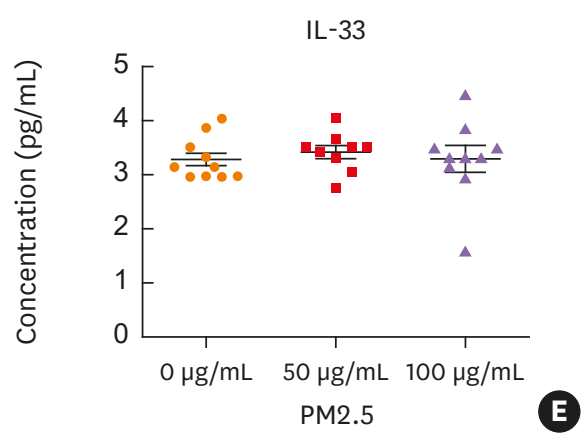

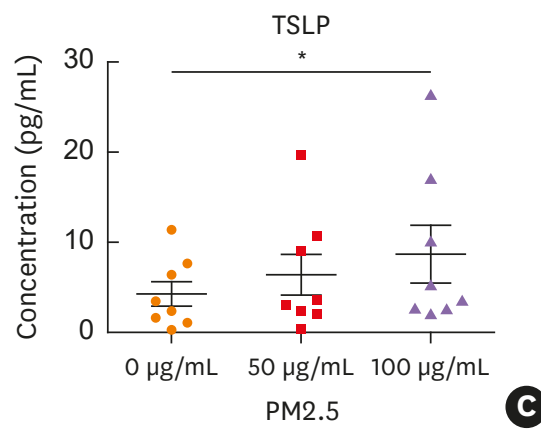

MMP-9

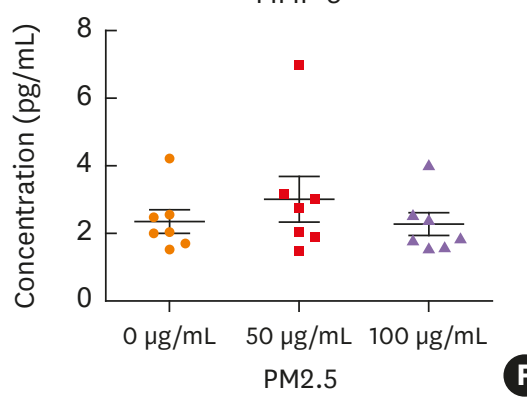

Fig. 6. Cytokine concentrations (pg/mL) in ALI culture medium of NECs treated with medium alone, $50 \mu \mathrm{g} / \mathrm{mL} \mathrm{PM} 2.5 \mathrm{or} 100 \mu \mathrm{gg} / \mathrm{mL} \mathrm{PM} 2.5$ intermittently for 3 days, respectively. Cultured NECs produced significantly more IL-8, TIMP-1 and TSLP after treatment with PM2.5, compared to cultures treated with medium alone. A dose-dependent effect was visible, although it was not statistically significant for all mediators. The secretion of other cytokines produced by NECs was not affected by PM2.5. Data are presented as mean \pm standard error of the mean ( $\mathrm{n}=8$ to 10 in each group).

PM2.5, particulate matter with an aerodynamic diameter less than $2.5 \mu \mathrm{m}$; IL, interleukin; TIMP-1, TIMP metallopeptidase inhibitor 1; TSLP, thymic stromal lymphopoietin; MMP, matrix metalloproteinase; NEC, nasal epithelial cell.

${ }^{*} P<0.05 ;{ }^{\dagger} P<0.01 ;{ }^{\ddagger} P<0.001$. 
Besides their role in constituting a barrier, epithelial cells are crucial in the initiation, regulation, and resolution of immune responses at mucosal sites..$^{34,35}$ To assess the effect of PM2.5 on the expression of immune responsive genes, we examined the cytokine/ chemokine production in NECs. Our study demonstrated that exposure of cultured NECs to PM2.5 led to increased production of IL-8, TIMP- 1 and TSLP, whereas the expressions of IL-25, IL-33, TGF- $\beta$ and MMP-9 were not affected. IL-8 production plays an important role in innate immunity involving neutrophil maturation, activation and chemotaxis. However, the enhanced immunity came at the price of remodeling and damaged barrier function. ${ }^{36} \mathrm{Yu}$ et al. ${ }^{37}$ also found that IL-8 regulated the permeability of endothelium by down-regulation of TJs in a dose- and time-dependence manner. Thus, the up-regulation of IL- 8 by PM2.5 is capable of reducing TJ proteins and leading to leaky epithelium. Our study has also indicated that although PM2.5 did not significantly alter the synthesis of MMP9, which has been shown to modulate TJ integrity and cell viability in human airway epithelia, ${ }^{38}$ it did significantly increase TIMP-1, an inhibitor of MMP9. The increased levels of TIMP-1 may contribute to airway remodeling. ${ }^{39}$ Bleck and colleagues ${ }^{40}$ had also shown that a wide variety of stimuli, including diesel-exhaust particles, can induce the human bronchial epithelial release of TSLP, which may profoundly affect allergen-specific IgE production/T helper 2 differentiation.

In conclusion, using NECs established as ALI cultures, we have demonstrated that exposure to PM2.5 leads to loss of the barrier integrity of the human nasal epithelium. It is likely that this may be attributed to a combination of decreased expression of TJ proteins and increased release of proinflammatory cytokines. These results suggest an important mechanism of susceptibility to nasal inflammation in areas with high levels of PM2.5 pollution. Because ambient PM2.5 concentrations in Beijing are much higher in the winter than in the summer, we speculate the PM2.5-induced decreases in TJ proteins and increased inflammation may be one of the factors contributing to increased respiratory problems in the winter months.

\section{ACKNOWLEDGMENTS}

This work was supported by grants from the national key R\&D program of China (2016YFC20160905200), the program for the Changjiang scholars and innovative research team (IRT13082), the national natural science foundation of China (81500771, 81470678, 81870698, 81420108009 and 81630023), Beijing municipal administration of hospitals' mission plan (SML20150203), and Beijing advanced innovation center for food nutrition and human health (Beijing Technology and Business University, 20181045). Beijing Municipal Administration of Hospitals Clinical Medicine Development of Special Funding Support (XMLX201816) and Capital Health Development Foundation (2016-1-2052). We thank the Institute for Environmental Health and Related Product Safety, Chinese Center for Disease Control and Prevention for providing the PM2.5 samples.

\section{SUPPLEMENTARY MATERIALS}

\section{Supplementary Data S1}

Supplementary document of PM2.5 causes deficiency in barrier integrity in human nasal epithelial cells. 


\section{Supplementary Table S1}

Content analysis of PM2.5 samples $\left(\mu \mathrm{g} / \mathrm{cm}^{2}\right)$

Click here to view

\section{Supplementary Table $\mathbf{S 2}$}

Primer sequences used for polymerase chain reaction (indicated as $5^{\prime}-3^{\prime}$ )

Click here to view

\section{Supplementary Table S3}

Clinical data of normal human nasal mucosa for immunohistochemistry evaluation of tight junctions

\section{Click here to view}

\section{Supplementary Table S4}

Cytokine concentrations $(\mathrm{pg} / \mathrm{mL})$ in air-liquid interface culture medium of nasal epithelial cells treated with medium alone, $50 \mu \mathrm{g} / \mathrm{mL}$ PM2.5 or $100 \mu \mathrm{g} / \mathrm{mL}$ PM2 2.5 intermittently for 3 days, respectively

Click here to view

\section{Supplementary Fig. S1}

Expression of tight junction mRNAs in noninflammatory nasal mucosa collected from different seasons. Arbitrary mRNA expression $\left(2^{-\triangle C T}\right.$ value $\left.\times 1,000\right)$ for ZO-1, ZO-2, claudin 1 , claudin 4 , claudin 7 and occludin of the winter samples tended to be lower than those of the summer samples, but none of the differences were statistically significant $(n=5$ each for the summer and winter).

Click here to view

\section{Supplementary Fig. $\mathbf{S 2}$}

Cytotoxicity of PM2.5 to cultured human NECs. PM2.5 was applied at concentrations ranging from $0.1 \mu \mathrm{g} / \mathrm{mL}$ to $1,000 \mu \mathrm{g} / \mathrm{mL}$ into medium of NECs culture. Culture supernatants were collected at 24 hours after addition of different doses of PM2.5 and lactate dehydrogenase release was measured. Data are expressed as mean \pm standard error of mean ( $\mathrm{n}=3$ different epithelial cells from healthy individuals per group). Experiments were performed in triplicate.

Click here to view

\section{Supplementary Fig. S3}

Recovery study of PM2.5's impact on barrier integrity in air-liquid interface cultures established from nasal epithelial cells. The relative TER of cultures were exposed to $100 \mu \mathrm{g} / \mathrm{mL}$ PM2.5 at 0, 24, 48 and 72 hours; after PM2.5 was withdrawn for 72 hours, 96 hours was shown. Data are expressed as mean \pm standard error of mean ( $n=3$ donors per group in duplicate). 


\section{REFERENCES}

1. Georas SN, Rezaee F. Epithelial barrier function: at the front line of asthma immunology and allergic airway inflammation. J Allergy Clin Immunol 2014;134:509-20.

PUBMED | CROSSREF

2. Soyka MB, Wawrzyniak P, Eiwegger T, Holzmann D, Treis A, Wanke K, et al. Defective epithelial barrier in chronic rhinosinusitis: the regulation of tight junctions by IFN- $\gamma$ and IL-4. J Allergy Clin Immunol 2012;130:1087-1096.e10. PUBMED | CROSSREF

3. Steelant B, Farré R, Wawrzyniak P, Belmans J, Dekimpe E, Vanheel H, et al. Impaired barrier function in patients with house dust mite-induced allergic rhinitis is accompanied by decreased occludin and zonula occludens-1 expression. J Allergy Clin Immunol 2016;137:1043-1053.e5. PUBMED | CROSSREF

4. Aghapour M, Raee P, Moghaddam SJ, Hiemstra PS, Heijink IH. Airway epithelial barrier dysfunction in chronic obstructive pulmonary disease: role of cigarette smoke exposure. Am J Respir Cell Mol Biol 2018;58:157-69. PUBMED | CROSSREF

5. Holgate ST. Mechanisms of asthma and implications for its prevention and treatment: a personal journey. Allergy Asthma Immunol Res 2013;5:343-7. PUBMED | CROSSREF

6. Darrow LA, Klein M, Flanders WD, Mulholland JA, Tolbert PE, Strickland MJ. Air pollution and acute respiratory infections among children 0-4 years of age: an 18-year time-series study. Am J Epidemiol 2014;180:968-77. PUBMED | CROSSREF

7. Feng C, Li J, Sun W, Zhang Y, Wang Q. Impact of ambient fine particulate matter (PM2.5) exposure on the risk of influenza-like-illness: a time-series analysis in Beijing, China. Environ Health 2016;15:17. PUBMED | CROSSREF

8. Bernstein DI. Diesel exhaust exposure, wheezing and sneezing. Allergy Asthma Immunol Res 2012;4:178-83. PUBMED | CROSSREF

9. Kumar RK, Shadie AM, Bucknall MP, Rutlidge H, Garthwaite L, Herbert C, et al. Differential injurious effects of ambient and traffic-derived particulate matter on airway epithelial cells. Respirology 2015;20:73-9. PUBMED | CROSSREF

10. Golebski K, Röschmann KI, Toppila-Salmi S, Hammad H, Lambrecht BN, Renkonen R, et al. The multifaceted role of allergen exposure to the local airway mucosa. Allergy 2013;68:152-60. PUBMED | CROSSREF

11. Sekiyama A, Gon Y, Terakado M, Takeshita I, Kozu Y, Maruoka S, et al. Glucocorticoids enhance airway epithelial barrier integrity. Int Immunopharmacol 2012;12:350-7. PUBMED | CROSSREF

12. Fujita H, Chalubinski M, Rhyner C, Indermitte P, Meyer N, Ferstl R, et al. Claudin-1 expression in airway smooth muscle exacerbates airway remodeling in asthmatic subjects. J Allergy Clin Immunol 2011;127:1612-1621.e8. PUBMED | CROSSREF

13. Lee JW, Hsiao WT, Chen HY, Hsu LP, Chen PR, Lin MD, et al. Upregulated claudin-1 expression confers resistance to cell death of nasopharyngeal carcinoma cells. Int J Cancer 2010;126:1353-66. PUBMED

14. Nair A, Vaidyanathan S, Clearie K, Williamson P, Meldrum K, Lipworth BJ. Steroid sparing effects of intranasal corticosteroids in asthma and allergic rhinitis. Allergy 2010;65:359-67. PUBMED | CROSSREF

15. Derendorf H, Meltzer EO. Molecular and clinical pharmacology of intranasal corticosteroids: clinical and therapeutic implications. Allergy 2008;63:1292-300. PUBMED | CROSSREF

16. Zhang Y, Lou H, Wang Y, Li Y, Zhang L, Wang C. Comparison of corticosteroids by 3 approaches to the treatment of chronic rhinosinusitis with nasal polyps. Allergy Asthma Immunol Res 2019;11:482-97. PUBMED | CROSSREF

17. Koizumi J, Kojima T, Kamekura R, Kurose M, Harimaya A, Murata M, et al. Changes of gap and tight junctions during differentiation of human nasal epithelial cells using primary human nasal epithelial cells and primary human nasal fibroblast cells in a noncontact coculture system. J Membr Biol 2007;218:1-7. PUBMED | CROSSREF 
18. Ohkuni T, Kojima T, Ogasawara N, Masaki T, Ninomiya T, Kikuchi S, et al. Expression and localization of tricellulin in human nasal epithelial cells in vivo and in vitro. Med Mol Morphol 2009;42:204-11. PUBMED | CROSSREF

19. Marchiando AM, Graham WV, Turner JR. Epithelial barriers in homeostasis and disease. Annu Rev Pathol 2010;5:119-44.

PUBMED | CROSSREF

20. Pan TL, Wang PW, Aljuffali IA, Huang CT, Lee CW, Fang JY. The impact of urban particulate pollution on skin barrier function and the subsequent drug absorption. J Dermatol Sci 2015;78:51-60. PUBMED | CROSSREF

21. Oppenheim HA, Lucero J, Guyot AC, Herbert LM, McDonald JD, Mabondzo A, et al. Exposure to vehicle emissions results in altered blood brain barrier permeability and expression of matrix metalloproteinases and tight junction proteins in mice. Part Fibre Toxicol 2013;10:62. PUBMED | CROSSREF

22. Wang T, Wang L, Moreno-Vinasco L, Lang GD, Siegler JH, Mathew B, et al. Particulate matter air pollution disrupts endothelial cell barrier via calpain-mediated tight junction protein degradation. Part Fibre Toxicol 2012;9:35. PUBMED | CROSSREF

23. Mutlu EA, Engen PA, Soberanes S, Urich D, Forsyth CB, Nigdelioglu R, et al. Particulate matter air pollution causes oxidant-mediated increase in gut permeability in mice. Part Fibre Toxicol 2011;8:19. PUBMED | CROSSREF

24. Liang Y, Fang L, Pan H, Zhang K, Kan H, Brook JR, et al. PM2.5 in Beijing - temporal pattern and its association with influenza. Environ Health 2014;13:102. PUBMED | CROSSREF

25. Kim DW, Chung SK, Na Y. Numerical study on the air conditioning characteristics of the human nasal cavity. Comput Biol Med 2017;86:18-30. PUBMED | CROSSREF

26. Heijink IH, Jonker MR, de Vries M, van Oosterhout AJ, Telenga E, Ten Hacken NH, et al. Budesonide and fluticasone propionate differentially affect the airway epithelial barrier. Respir Res 2016;17:2 PUBMED | CROSSREF

27. Fischer A, Gluth M, Weege F, Pape UF, Wiedenmann B, Baumgart DC, et al. Glucocorticoids regulate barrier function and claudin expression in intestinal epithelial cells via MKP-1. Am J Physiol Gastrointest Liver Physiol 2014;306:G218-28. PUBMED | CROSSREF

28. Schamberger AC, Mise N, Jia J, Genoyer E, Yildirim AÖ, Meiners S, et al. Cigarette smoke-induced disruption of bronchial epithelial tight junctions is prevented by transforming growth factor- $\beta$. Am J Respir Cell Mol Biol 2014;50:1040-52. PUBMED | CROSSREF

29. Henriquez OA, Den Beste K, Hoddeson EK, Parkos CA, Nusrat A, Wise SK. House dust mite allergen Der p 1 effects on sinonasal epithelial tight junctions. Int Forum Allergy Rhinol 2013;3:630-5. PUBMED | CROSSREF

30. Fukuoka A, Matsushita K, Morikawa T, Takano H, Yoshimoto T. Diesel exhaust particles exacerbate allergic rhinitis in mice by disrupting the nasal epithelial barrier. Clin Exp Allergy 2016;46:142-52. PUBMED | CROSSREF

31. Rogers GA, Den Beste K, Parkos CA, Nusrat A, Delgaudio JM, Wise SK. Epithelial tight junction alterations in nasal polyposis. Int Forum Allergy Rhinol 2011;1:50-4. PUBMED | CROSSREF

32. Altunbulakli C, Costa R, Lan F, Zhang N, Akdis M, Bachert C, et al. Staphylococcus aureus enhances the tight junction barrier integrity in healthy nasal tissue, but not in nasal polyps. J Allergy Clin Immunol 2018;142:665-668.e8. PUBMED | CROSSREF

33. Zhang Y, Lan F, Li Y, Wang C, Zhang L. Formation of papillary mucosa folds and enhancement of epithelial barrier in odontogenic sinusitis. Int Forum Allergy Rhinol. Forthcoming 2019. PUBMED | CROSSREF

34. Saenz SA, Taylor BC, Artis D. Welcome to the neighborhood: epithelial cell-derived cytokines license innate and adaptive immune responses at mucosal sites. Immunol Rev 2008;226:172-90. PUBMED | CROSSREF

35. Oboki K, Nakae S, Matsumoto K, Saito H. IL-33 and airway inflammation. Allergy Asthma Immunol Res 2011;3:81-8.

PUBMED | CROSSREF 
36. Reynolds CJ, Quigley K, Cheng X, Suresh A, Tahir S, Ahmed-Jushuf F, et al. Lung defense through IL-8 carries a cost of chronic lung remodeling and impaired function. Am J Respir Cell Mol Biol 2018;59:557-71. PUBMED | CROSSREF

37. Yu H, Huang X, Ma Y, Gao M, Wang O, Gao T, et al. Interleukin-8 regulates endothelial permeability by down-regulation of tight junction but not dependent on integrins induced focal adhesions. Int J Biol Sci 2013;9:966-79.

PUBMED | CROSSREF

38. Vermeer PD, Denker J, Estin M, Moninger TO, Keshavjee S, Karp P, et al. MMP9 modulates tight junction integrity and cell viability in human airway epithelia. Am J Physiol Lung Cell Mol Physiol 2009;296:L751-62. PUBMED | CROSSREF

39. Park CS, Kim TB, Moon KA, Bae YJ, Lee HR, Jang MK, et al. Chlamydophila pneumoniae enhances secretion of VEGF, TGF-beta and TIMP-1 from human bronchial epithelial cells under Th2 dominant microenvironment. Allergy Asthma Immunol Res 2010;2:41-7. PUBMED | CROSSREF

40. Bleck B, Tse DB, Gordon T, Ahsan MR, Reibman J. Diesel exhaust particle-treated human bronchial epithelial cells upregulate Jagged- 1 and OX40 ligand in myeloid dendritic cells via thymic stromal lymphopoietin. J Immunol 2010;185:6636-45.

PUBMED | CROSSREF 